\title{
Region of variability for functions with positive real part
}

\author{
by Saminathan Ponnusamy and Allu Vasudevarao (Chennai)
}

\begin{abstract}
For $\gamma \in \mathbb{C}$ such that $|\gamma|<\pi / 2$ and $0 \leq \beta<1$, let $\mathcal{P}_{\gamma, \beta}$ denote the class of all analytic functions $P$ in the unit disk $\mathbb{D}$ with $P(0)=1$ and

$$
\operatorname{Re}\left(e^{i \gamma} P(z)\right)>\beta \cos \gamma \quad \text { in } \mathbb{D} .
$$

For any fixed $z_{0} \in \mathbb{D}$ and $\lambda \in \overline{\mathbb{D}}$, we shall determine the region of variability $V_{\mathcal{P}}\left(z_{0}, \lambda\right)$ for $\int_{0}^{z_{0}} P(\zeta) d \zeta$ when $P$ ranges over the class

$$
\mathcal{P}(\lambda)=\left\{P \in \mathcal{P}_{\gamma, \beta}: P^{\prime}(0)=2(1-\beta) \lambda e^{-i \gamma} \cos \gamma\right\} .
$$

As a consequence, we present the region of variability for some subclasses of univalent functions. We also graphically illustrate the region of variability for several sets of parameters.
\end{abstract}

1. Introduction. We denote by $\mathcal{H}$ the class of analytic functions in the unit disk $\mathbb{D}=\{z \in \mathbb{C}:|z|<1\}$, and think of $\mathcal{H}$ as a topological vector space endowed with the topology of uniform convergence over compact subsets of $\mathbb{D}$. We consider the subclass of functions $\phi \in \mathcal{H}$ with $\phi(0)=0=\phi^{\prime}(0)-1$ such that $\phi$ maps $\mathbb{D}$ univalently onto a domain that is starlike (with respect to the origin). That is, $t \phi(z) \in \phi(\mathbb{D})$ for each $t \in[0,1]$. We denote the class of such functions by $\mathcal{S}^{*}$. Analytically, each $\phi \in \mathcal{S}^{*}$ is characterized by the condition

$$
\operatorname{Re}\left(\frac{z \phi^{\prime}(z)}{\phi(z)}\right)>0, \quad z \in \mathbb{D} .
$$

Functions in $\mathcal{S}^{*}$ are referred to as starlike functions. A function $\phi \in \mathcal{H}$ with $\phi(0)=0=\phi^{\prime}(0)-1$ is said to belong to $\mathcal{C}$ if and only if $\phi(\mathbb{D})$ is a convex domain. It is well-known that $\phi \in \mathcal{C}$ if and only if $z \phi^{\prime} \in \mathcal{S}^{*}$. Functions in $\mathcal{C}$ are referred to as convex functions.

Let $\mathcal{P}_{\gamma, \beta}$ denote the class of functions $P \in \mathcal{H}$ with $P(0)=1$ and

$$
\operatorname{Re}\left(e^{i \gamma} P(z)\right)>\beta \cos \gamma \quad \text { in } \mathbb{D}
$$

2010 Mathematics Subject Classification: Primary 30C45; Secondary 30C55,30C80.

Key words and phrases: analytic, univalent, starlike and convex functions, Schwarz lemma, convolution, variability region. 
for some $\beta$ with $\beta<1$ and $\gamma \in \mathbb{C}$ with $|\gamma|<\pi / 2$. Let $\mathcal{A}$ denote the class of functions $f$ in $\mathcal{H}$ such that $f(0)=0=f^{\prime}(0)-1$. When $P(z)=z f^{\prime}(z) / f(z)$ and $\beta=0$, the class $\mathcal{P}_{\gamma, \beta}$ becomes

$$
\mathcal{S}^{\gamma}(0)=\left\{f \in \mathcal{A}: \operatorname{Re}\left(e^{i \gamma} \frac{z f^{\prime}(z)}{f(z)}\right)>0 \text { in } \mathbb{D}\right\}
$$

for some $\gamma$ with $|\gamma|<\pi / 2$. Functions in $\mathcal{S}^{\gamma}(0)$ are known to be univalent in $\mathbb{D}$ and $\mathcal{S}^{0}(0) \equiv \mathcal{S}^{*}$. Functions in $\mathcal{S}^{\gamma}(0)$ are called spirallike functions (see [S]).

2. Preliminary investigation about the class $\mathcal{P}_{\gamma, \beta}$. Herglotz representation for analytic functions with positive real part in $\mathbb{D}$ shows that if $P \in \mathcal{P}_{\gamma, \beta}$, then there exists a unique positive unit measure $\mu$ on $(-\pi, \pi]$ such that

$$
P(z)=\int_{-\pi}^{\pi} \frac{1+\left[1-2 \beta e^{-i \gamma} \cos \gamma\right] z e^{-i t}}{1-z e^{-i t}} d \mu(t) .
$$

Let $\mathcal{B}_{0}$ be the class of analytic functions $\omega$ in $\mathbb{D}$ such that $|\omega(z)|<1$ in $\mathbb{D}$ and $\omega(0)=0$. Then it is a simple exercise to see that for each $P \in \mathcal{P}_{\gamma, \beta}$ there exists an $\omega_{P} \in \mathcal{B}_{0}$ such that

$$
\omega_{P}(z)=\frac{e^{i \gamma} P(z)-e^{i \gamma}}{e^{i \gamma} P(z)-\left(2 \beta \cos \gamma-e^{-i \gamma}\right)}, \quad z \in \mathbb{D},
$$

and conversely. Clearly, we have

$$
P^{\prime}(0)=2 e^{-i \gamma} \omega_{P}^{\prime}(0)(1-\beta) \cos \gamma .
$$

Suppose that $P \in \mathcal{P}_{\gamma, \beta}$. Then, because $\left|\omega_{P}^{\prime}(0)\right| \leq 1$, by the classical Schwarz lemma (see for example [Di, Du, $\mathrm{Po2}, \mathrm{PS}]$ ) we may let

$$
P^{\prime}(0)=2 \lambda e^{-i \gamma}(1-\beta) \cos \gamma
$$

for some $\lambda \in \overline{\mathbb{D}}$, with $\omega_{P}^{\prime}(0)=\lambda$. Using 2.1), one can compute

$$
\frac{\omega_{P}^{\prime \prime}(0)}{2}=\frac{e^{i \gamma} P^{\prime \prime}(0)}{4(1-\beta) \cos \gamma}-\lambda^{2} \text {. }
$$

Also if we let

$$
g(z)= \begin{cases}\frac{\omega_{P}(z) / z-\lambda}{1-\bar{\lambda} \omega_{P}(z) / z} & \text { for }|\lambda|<1 \\ 0 & \text { for }|\lambda|=1\end{cases}
$$

then we see that

$$
g^{\prime}(0)= \begin{cases}\left.\frac{1}{1-|\lambda|^{2}}\left(\frac{\omega_{P}(z)}{z}\right)^{\prime}\right|_{z=0}=\frac{1}{1-|\lambda|^{2}} \frac{\omega_{P}^{\prime \prime}(0)}{2} & \text { for }|\lambda|<1, \\ 0 & \text { for }|\lambda|=1 .\end{cases}
$$


By the Schwarz lemma, $|g(z)| \leq|z|$ and $\left|g^{\prime}(0)\right| \leq 1$. Equality holds in both the cases if and only if $g(z)=e^{i \alpha} z$ for some $\alpha \in \mathbb{R}$. The condition $\left|g^{\prime}(0)\right| \leq 1$ shows that there exists an $a \in \overline{\mathbb{D}}$ such that $g^{\prime}(0)=a$.

In view of 2.2 we may represent $P^{\prime \prime}(0)$ as

$$
P^{\prime \prime}(0)=4(1-\beta)\left[\left(1-|\lambda|^{2}\right) a+\lambda^{2}\right] e^{-i \gamma} \cos \gamma
$$

for some $a \in \overline{\mathbb{D}}$. Consequently, for $\lambda \in \overline{\mathbb{D}}=\{z \in \mathbb{C}:|z| \leq 1\}$ and $z_{0} \in \mathbb{D}$ fixed, it is natural to introduce (for convenience with the notation $\mathcal{P}(\lambda)$ instead of $\left.\mathcal{P}_{\gamma, \beta}(\lambda)\right)$

$$
\begin{aligned}
\mathcal{P}(\lambda) & =\left\{P \in \mathcal{P}_{\gamma, \beta}: P^{\prime}(0)=2(1-\beta) e^{-i \gamma} \lambda \cos \gamma\right\}, \\
V_{\mathcal{P}}\left(z_{0}, \lambda\right) & =\left\{\int_{0}^{z_{0}} P(\zeta) d \zeta: P \in \mathcal{P}(\lambda)\right\} .
\end{aligned}
$$

Obviously, each $f \in \mathcal{P}(\lambda)$ has to satisfy condition $(2.3)$ for some $a \in \overline{\mathbb{D}}$ and so we do not need to include it in the definition of $\mathcal{P}(\lambda)$.

For each fixed $z_{0} \in \mathbb{D}$, using extreme function theory, it has been shown by Grunsky [Du, Theorem 10.6] that the region of variability of

$$
V\left(z_{0}\right)=\left\{\log \frac{f\left(z_{0}\right)}{z_{0}}: f \in \mathcal{S}\right\}
$$

is precisely a closed disk, where $\mathcal{S}=\{f \in \mathcal{A}: f$ is univalent in $\mathbb{D}\}$. It is also well-known that the region of variability

$$
V\left(z_{0}\right)=\left\{\log \phi^{\prime}\left(z_{0}\right): \phi \in \mathcal{C}\right\}
$$

is the set $\left\{\log (1-z)^{-2}:|z| \leq\left|z_{0}\right|\right\}$. Several authors have studied region of variability problems for various subclasses of univalent functions in $\mathcal{H}$; see [Pa, Pi, PV, PVV1, PVV2, PVY1, PVY2, Y1, YY2.

The main aim of this paper is to determine the region of variability of $V_{\mathcal{P}}\left(z_{0}, \lambda\right)$ for $\int_{0}^{z_{0}} P(\zeta) d \zeta$ when $P$ ranges over the class $\mathcal{P}(\lambda)$. In Section 3 . we present some basic properties of $V_{\mathcal{P}}\left(z_{0}, \lambda\right)$, whereas in Section 4 , we investigate the growth condition for functions in $\mathcal{P}(\lambda)$. The precise geometric description of the set $V_{\mathcal{P}}\left(z_{0}, \lambda\right)$ is established in Theorem 5.1 in Section 5 . Two interesting special cases are presented in Section 6. Finally, in Section 7 , we graphically represent the region of variability for several sets of parameters.

3. Basic properties of $V_{\mathcal{P}}\left(z_{0}, \lambda\right)$. For a positive integer $p$, let $\left(\mathcal{S}^{*}\right)^{p}=$ $\left\{f=f_{0}^{p}: f_{0} \in \mathcal{S}^{*}\right\}$. A sufficient condition (see [Y1]) for an analytic function $f$ in $\mathbb{D}$ with $f(z)=z^{p}+\cdots$ to be in $\left(\mathcal{S}^{*}\right)^{p}$ is

$$
\operatorname{Re}\left(1+z \frac{f^{\prime \prime}(z)}{f^{\prime}(z)}\right)>0, \quad z \in \mathbb{D} .
$$

This fact will be used in the following result. 
Proposition 3.1.

(1) $V_{\mathcal{P}}\left(z_{0}, \lambda\right)$ is a compact subset of $\mathbb{C}$.

(2) $V_{\mathcal{P}}\left(z_{0}, \lambda\right)$ is a convex subset of $\mathbb{C}$.

(3) For $|\lambda|=1$ or $z_{0}=0$,

$$
V_{\mathcal{P}}\left(z_{0}, \lambda\right)=\left\{z_{0}-2(1-\beta) e^{-i \gamma}(\cos \gamma)\left(z_{0}+\frac{1}{\lambda} \log \left(1-\lambda z_{0}\right)\right)\right\} .
$$

(4) For $|\lambda|<1$ and $z_{0} \in \mathbb{D} \backslash\{0\}, V_{\mathcal{P}}\left(z_{0}, \lambda\right)$ has

$$
z_{0}-2(1-\beta) e^{-i \gamma}(\cos \gamma)\left(z_{0}+\frac{1}{\lambda} \log \left(1-\lambda z_{0}\right)\right)
$$

as an interior point.

Proof. (1) Since $\mathcal{P}(\lambda)$ is a compact subset of $\mathcal{H}$, it follows that $V_{\mathcal{P}}\left(z_{0}, \lambda\right)$ is also compact.

(2) If $p_{1}, p_{2} \in \mathcal{P}(\lambda)$ and $0 \leq t \leq 1$, then the function

$$
P_{t}(z)=(1-t) p_{1}(z)+t p_{2}(z)
$$

is evidently in $\mathcal{P}(\lambda)$. Also, because of the representation of $P_{t}$, we easily see that the set $V_{\mathcal{P}}\left(z_{0}, \lambda\right)$ is convex.

(3) If $z_{0}=0,(3.2)$ trivially holds. If $|\lambda|=1$, then from our earlier observation $\omega_{P}(z)=\lambda z$, and so $P \in \mathcal{P}(\lambda)$ defined by 2.1) takes the form

$$
P(z)=\frac{1+\lambda z\left[2(1-\beta) e^{-i \gamma} \cos \gamma-1\right]}{1-\lambda z},
$$

or equivalently,

$$
P(z)=1-2(1-\beta) e^{-i \gamma}(\cos \gamma)\left(1-\frac{1}{1-\lambda z}\right) .
$$

Consequently,

$$
V_{\mathcal{P}}\left(z_{0}, \lambda\right)=\left\{z_{0}-2(1-\beta) e^{-i \gamma}(\cos \gamma)\left(z_{0}+\frac{1}{\lambda} \log \left(1-\lambda z_{0}\right)\right)\right\} .
$$

(4) For $|\lambda|<1$ and $a \in \overline{\mathbb{D}}$, we let

$$
\delta(z, \lambda)=\frac{z+\lambda}{1+\bar{\lambda} z}
$$

and in order to get the extremal function in $\mathcal{P}(\lambda)$, we define

$$
H_{a, \lambda}(z)=1+2(1-\beta) e^{-i \gamma} \cos \gamma \frac{\delta(a z, \lambda) z}{1-\delta(a z, \lambda) z} .
$$

Clearly $H_{a, \lambda}(0)=1$. Since $\delta(a z, \lambda)$ lies in the unit disk $\mathbb{D}$ and $\varphi(w)=$ $w /(1-w)$ maps $|w|<1$ onto $\operatorname{Re} \varphi(w)>-1 / 2$, we obtain

$$
\operatorname{Re}\left(e^{i \gamma} H_{a, \lambda}(z)\right)>\beta \cos \gamma \quad \text { in } \mathbb{D} \text {. }
$$


Also, from (3.4), we have the normalization condition

$$
H_{a, \lambda}^{\prime}(0)=2(1-\beta) e^{-i \gamma} \lambda \cos \gamma .
$$

Thus, $H_{a, \lambda} \in \mathcal{P}(\lambda)$. We observe that

$$
\omega_{H_{a, \lambda}}(z)=z \delta(a z, \lambda) .
$$

We claim that the mapping

$$
\mathbb{D} \ni a \mapsto \int_{0}^{z_{0}} H_{a, \lambda}(\zeta) d \zeta
$$

is a non-constant analytic function of $a$ for each fixed $z_{0} \in \mathbb{D} \backslash\{0\}$ and $\lambda \in \mathbb{D}$. To see this, we introduce

$$
h(z)=\left.\frac{3 e^{i \gamma}}{2(1-\beta)\left(1-|\lambda|^{2}\right) \cos \gamma} \frac{\partial}{\partial a}\left\{\int_{0}^{z} H_{a, \lambda}(\zeta) d \zeta\right\}\right|_{a=0}
$$

so that

$$
h(z)=\left.\frac{3}{1-|\lambda|^{2}} \frac{\partial}{\partial a}\left\{\int_{0}^{z} \frac{\delta(a \zeta, \lambda) \zeta}{1-\delta(a \zeta, \lambda) \zeta} d \zeta\right\}\right|_{a=0} .
$$

A computation gives

$$
h(z)=\left.3 \frac{\partial}{\partial a}\left\{\int_{0}^{z} \frac{\zeta^{2}}{(1-\lambda \zeta)^{2}} \frac{d \zeta}{(1-a \delta(a \zeta, \lambda) \zeta)^{2}}\right\}\right|_{a=0},
$$

which clearly implies

$$
h(z)=3 \int_{0}^{z} \frac{\zeta^{2}}{(1-\lambda \zeta)^{2}} d \zeta=z^{3}+\cdots
$$

from which it is easy to see that

$$
\operatorname{Re}\left\{\frac{z h^{\prime \prime}(z)}{h^{\prime}(z)}\right\}=2 \operatorname{Re}\left\{\frac{1}{1-\lambda z}\right\}>\frac{2}{1+|\lambda|} \geq 1, \quad z \in \mathbb{D} .
$$

By (3.1), there exists a function $h_{0} \in \mathcal{S}^{*}$ with $h=h_{0}^{3}$. The univalence of $h_{0}$ together with the condition $h_{0}(0)=0$ implies that $h\left(z_{0}\right) \neq 0$ for $z_{0} \in \mathbb{D} \backslash\{0\}$. Consequently, the mapping $\mathbb{D} \ni a \mapsto \int_{0}^{z_{0}} H_{a, \lambda}(\zeta) d \zeta$ is a nonconstant analytic function of $a$, and hence it is an open mapping. Thus, $V_{\mathcal{P}}\left(z_{0}, \lambda\right)$ contains the open set

$$
\left\{\int_{0}^{z_{0}} H_{a, \lambda}(\zeta) d \zeta:|a|<1\right\}
$$

In particular,

$$
\int_{0}^{z_{0}} H_{0, \lambda}(\zeta) d \zeta=z_{0}-2(1-\beta) e^{-i \gamma}(\cos \gamma)\left(z_{0}+\frac{1}{\lambda} \log \left(1-\lambda z_{0}\right)\right)
$$


is an interior point of

$$
\left\{\int_{0}^{z_{0}} H_{a, \lambda}(\zeta) d \zeta: a \in \mathbb{D}\right\} \subset V_{\mathcal{P}}\left(z_{0}, \lambda\right) .
$$

We remark that, since $V_{\mathcal{P}}\left(z_{0}, \lambda\right)$ is a compact convex subset of $\mathbb{C}$ and has non-empty interior, the boundary $\partial V_{\mathcal{P}}\left(z_{0}, \lambda\right)$ is a Jordan curve and $V_{\mathcal{P}}\left(z_{0}, \lambda\right)$ is the union of $\partial V_{\mathcal{P}}\left(z_{0}, \lambda\right)$ and its inner domain.

\section{Growth condition for functions in $\mathcal{P}(\lambda)$}

Proposition 4.1. For $P \in \mathcal{P}(\lambda)$ with $\lambda \in \mathbb{D}$, we have

$$
|P(z)-c(z, \lambda)| \leq r(z, \lambda), \quad z \in \mathbb{D},
$$

where

$$
\begin{aligned}
c(z, \lambda)= & \frac{\left(1+\lambda z\left(e^{-i \gamma}-2 \beta \cos \gamma\right) e^{-i \gamma}\right)(1-\bar{\lambda} \bar{z})}{\left(1-|z|^{2}\right)\left(1+|z|^{2}-2 \operatorname{Re}(\lambda z)\right)} \\
& +\frac{|z|^{2}(\bar{z}-\lambda)\left(\bar{\lambda}+z\left(e^{-i \gamma}-2 \beta \cos \gamma\right) e^{-i \gamma}\right)}{\left(1-|z|^{2}\right)\left(1+|z|^{2}-2 \operatorname{Re}(\lambda z)\right)}, \\
r(z, \lambda)= & \frac{2\left(1-|\lambda|^{2}\right)(1-\beta)|z|^{2} \cos \gamma}{\left(1-|z|^{2}\right)\left(1+|z|^{2}-2 \operatorname{Re}(\lambda z)\right)} .
\end{aligned}
$$

For each $z \in \mathbb{D} \backslash\{0\}$, equality holds if and only if $P=H_{e^{i \theta}, \lambda}$ for some $\theta \in \mathbb{R}$.

Proof. Let $P \in \mathcal{P}(\lambda)$. Then there exists $\omega_{P} \in \mathcal{B}_{0}$ satisfying (2.1). As observed in Section $2(|g(z)| \leq|z|)$, we have

$$
\left|\frac{\omega_{P}(z) / z-\lambda}{1-\bar{\lambda} \omega_{P}(z) / z}\right| \leq|z|, \quad z \in \mathbb{D} .
$$

From (2.1) this is equivalent to

$$
\left|\frac{P(z)-A(z, \lambda)}{P(z)+B(z, \lambda)}\right| \leq|z||\tau(z, \lambda)|
$$

where

$$
\left\{\begin{array}{l}
A(z, \lambda)=\frac{1+e^{-i \gamma} \lambda z\left(e^{-i \gamma}-2 \beta \cos \gamma\right)}{1-\lambda z} \\
B(z, \lambda)=\frac{\bar{\lambda}+e^{-i \gamma} z\left(e^{-i \gamma}-2 \beta \cos \gamma\right)}{z-\bar{\lambda}} \\
\tau(z, \lambda)=\frac{z-\bar{\lambda}}{1-\lambda z}
\end{array}\right.
$$


A simple calculation shows that the inequality 4.3 is equivalent to

$$
\begin{aligned}
\left|P(z)-\frac{A(z, \lambda)+|z|^{2}|\tau(z, \lambda)|^{2} B(z, \lambda)}{1-|z|^{2}|\tau(z, \lambda)|^{2}}\right| & \\
\leq & \frac{|z||\tau(z, \lambda)||A(z, \lambda)+B(z, \lambda)|}{1-|z|^{2}|\tau(z, \lambda)|^{2}} .
\end{aligned}
$$

Using (4.4) we can easily see that

$$
\begin{aligned}
& 1-|z|^{2}|\tau(z, \lambda)|^{2}=\frac{\left(1-|z|^{2}\right)\left(1+|z|^{2}-2 \operatorname{Re}(\lambda z)\right)}{|1-\lambda z|^{2}}, \\
& A(z, \lambda)+B(z, \lambda)=\frac{2\left(1-|\lambda|^{2}\right)(1-\beta)(\cos \gamma) e^{-i \gamma} z}{(1-\lambda z)(z-\bar{\lambda})}
\end{aligned}
$$

and

$$
\begin{aligned}
A(z, \lambda)+|z|^{2}|\tau(z, \lambda)|^{2} B(z, \lambda) & \\
= & \frac{\left(1+\lambda z\left(e^{-i \gamma}-2 \beta \cos \gamma\right) e^{-i \gamma}\right)(1-\bar{\lambda} \bar{z})}{|1-\lambda z|^{2}} \\
& +\frac{|z|^{2}(\bar{z}-\lambda)\left(\bar{\lambda}+z\left(e^{-i \gamma}-2 \beta \cos \gamma\right) e^{-i \gamma}\right)}{|1-\lambda z|^{2}} .
\end{aligned}
$$

Thus, by a simple computation, we see that

$$
\begin{aligned}
& \frac{A(z, \lambda)+|z|^{2}|\tau(z, \lambda)|^{2} B(z, \lambda)}{1-|z|^{2}|\tau(z, \lambda)|^{2}}=c(z, \lambda), \\
& \frac{|z||\tau(z, \lambda)||A(z, \lambda)+B(z, \lambda)|}{1-|z|^{2}|\tau(z, \lambda)|^{2}}=r(z, \lambda) .
\end{aligned}
$$

Now the inequality 4.1 follows from these equalities and 4.5).

It is easy to see that equality occurs in 4.1 for a $z \in \mathbb{D}$ when $P=H_{e^{i \theta}, \lambda}$ for some $\theta \in \mathbb{R}$. Conversely if equality occurs in (4.1) for some $z \in \mathbb{D} \backslash\{0\}$, then equality must hold in (4.2). Thus from the Schwarz lemma there exists a $\theta \in \mathbb{R}$ such that $\omega_{P}(z)=z \delta\left(e^{i \theta} z, \lambda\right)$ for all $z \in \mathbb{D}$. This implies $P=H_{e^{i \theta}, \lambda}$.

The choice of $\lambda=0$ gives the following result which may deserve a special mention.

Corollary 4.2. For $P \in \mathcal{P}(0)$ we have

$$
\left|P(z)-\frac{1+(1-2 \beta)|z|^{4}}{1-|z|^{4}}\right| \leq \frac{2(1-\beta)|z|^{2}}{1-|z|^{4}}, \quad z \in \mathbb{D} .
$$

For each $z \in \mathbb{D} \backslash\{0\}$, equality holds if and only if $P=H_{e^{i \theta}, 0}$ for some $\theta \in \mathbb{R}$. 
Corollary 4.3. Let $\gamma: z(t), 0 \leq t \leq 1$, be a $C^{1}$-curve in $\mathbb{D}$ with $z(0)=0$ and $z(1)=z_{0}$. Then

$$
V_{\mathcal{P}}\left(z_{0}, \lambda\right) \subset\{w \in \mathbb{C}:|w-C(\lambda, \gamma)| \leq R(\lambda, \gamma)\},
$$

where

$$
C(\lambda, \gamma)=\int_{0}^{1} c(z(t), \lambda) z^{\prime}(t) d t \quad \text { and } \quad R(\lambda, \gamma)=\int_{0}^{1} r(z(t), \lambda)\left|z^{\prime}(t)\right| d t
$$

Proof. The proof follows as in PVV2].

For the proof of our next result, we need the following lemma.

Lemma 4.4. For $\theta \in \mathbb{R}$ and $\lambda \in \mathbb{D}$, the function

$$
G(z)=\int_{0}^{z} \frac{e^{i \theta} \zeta^{2}}{\left\{1+\left(\bar{\lambda} e^{i \theta}-\lambda\right) \zeta-e^{i \theta} \zeta^{2}\right\}^{2}} d \zeta, \quad z \in \mathbb{D},
$$

has a zero of order three at the origin and no zeros elsewhere in $\mathbb{D}$. Furthermore there exists a starlike univalent function $G_{0}$ in $\mathbb{D}$ such that $G=\frac{1}{3} e^{i \theta} G_{0}^{3}$ and $G_{0}(0)=G_{0}^{\prime}(0)-1=0$.

Proof. For a proof, we refer to [PVV2, Lemma 3.4] with $\beta=1$ there.

Proposition 4.5. Let $z_{0} \in \mathbb{D} \backslash\{0\}$. Then for $\theta \in(-\pi, \pi]$ we have

$$
\int_{0}^{z_{0}} H_{e^{i \theta}, \lambda}(\zeta) d \zeta \in \partial V_{\mathcal{P}}\left(z_{0}, \lambda\right)
$$

Furthermore if $\int_{0}^{z_{0}} P(\zeta) d \zeta=\int_{0}^{z_{0}} H_{e^{i \theta}, \lambda}(\zeta) d \zeta$ for some $P \in \mathcal{P}(\lambda)$ and $\theta \in$ $(-\pi, \pi]$, then $P=H_{e^{i \theta}, \lambda}$.

Proof. From (3.4) we have

$$
\begin{aligned}
H_{a, \lambda}(z) & =\frac{1+\left[2(1-\beta)(\cos \gamma) e^{-i \gamma}-1\right] \delta(a z, \lambda) z}{1-\delta(a z, \lambda) z} \\
& =\frac{1+\bar{\lambda} a z+\left(\lambda z+a z^{2}\right)\left(2(1-\beta)(\cos \gamma) e^{-i \gamma}-1\right)}{1+(\bar{\lambda} a-\lambda) z-a z^{2}} .
\end{aligned}
$$

Using (4.4) we compute

$$
\begin{aligned}
& H_{a, \lambda}(z)-A(z, \lambda)=\frac{2(1-\beta)\left(1-|\lambda|^{2}\right)(\cos \gamma) e^{-i \gamma} a z^{2}}{(1-\lambda z)\left(1+(\bar{\lambda} a-\lambda) z-a z^{2}\right)} \\
& H_{a, \lambda}(z)+B(z, \lambda)=\frac{2(1-\beta)\left(1-|\lambda|^{2}\right)(\cos \gamma) e^{-i \gamma} z}{(z-\bar{\lambda})\left(1+(\bar{\lambda} a-\lambda) z-a z^{2}\right)}
\end{aligned}
$$


and hence

$$
\begin{aligned}
H_{a, \lambda}(z)-c(z, \lambda)= & H_{a, \lambda}(z)-\frac{A(z, \lambda)+|z|^{2}|\tau(z, \lambda)|^{2} B(z, \lambda)}{1-|z|^{2}|\tau(z, \lambda)|^{2}} \\
= & \frac{1}{1-|z|^{2}|\tau(z, \lambda)|^{2}}\left\{\begin{array}{c}
H_{a, \lambda}(z)-A(z, \lambda) \\
\left.\quad-|z|^{2}|\tau(z, \lambda)|^{2}\left(H_{a, \lambda}(z)+B(z, \lambda)\right)\right\}
\end{array}\right. \\
= & \frac{2(1-\beta)\left(1-|\lambda|^{2}\right)(\cos \gamma) e^{-i \gamma} a z^{2}\left[\overline{\left.1+(\bar{\lambda} a-\lambda) z-a z^{2}\right]}\right.}{\left(1-|z|^{2}\right)\left(1+|z|^{2}-2 \operatorname{Re}(\lambda z)\right)\left(1+(\bar{\lambda} a-\lambda) z-a z^{2}\right)} \\
= & r(z, \lambda) \frac{e^{-i \gamma} a z^{2}}{|z|^{2}} \frac{\left|1+(\bar{\lambda} a-\lambda) z-a z^{2}\right|^{2}}{\left(1+(\bar{\lambda} a-\lambda) z-a z^{2}\right)^{2}} .
\end{aligned}
$$

Now by substituting $a=e^{i \theta}$ we easily see that

$$
H_{e^{i \theta}, \lambda}(z)-c(z, \lambda)=r(z, \lambda) \frac{e^{-i \gamma} e^{i \theta} z^{2}}{|z|^{2}} \frac{\left|1+\left(\bar{\lambda} e^{i \theta}-\lambda\right) z-e^{i \theta} z^{2}\right|^{2}}{\left(1+\left(\bar{\lambda} e^{i \theta}-\lambda\right) z-e^{i \theta} z^{2}\right)^{2}} .
$$

For $G(z)$ as in Lemma 4.4 , we get

$$
H_{e^{i \theta}, \lambda}(z)-c(z, \lambda)=r(z, \lambda) e^{-i \gamma} \frac{G^{\prime}(z)}{\left|G^{\prime}(z)\right|}
$$

and there exists a starlike univalent function $G_{0}$ in $\mathbb{D}$ such that $G=$ $\frac{1}{3} e^{i \theta} G_{0}^{3}$ and $G_{0}(0)=G_{0}^{\prime}(0)-1=0$. As $G_{0}$ is starlike, for any $z_{0} \in \mathbb{D} \backslash\{0\}$ the linear segment joining 0 and $G_{0}\left(z_{0}\right)$ entirely lies in $G_{0}(\mathbb{D})$.

Now, we define $\gamma_{0}$ by

$$
\gamma_{0}: z(t)=G_{0}^{-1}\left(t G_{0}\left(z_{0}\right)\right), \quad 0 \leq t \leq 1 .
$$

Since $G(z(t))=\frac{1}{3} e^{i \theta}\left(G_{0}(z(t))\right)^{3}=\frac{1}{3} e^{i \theta}\left(t G_{0}\left(z_{0}\right)\right)^{3}=t^{3} G\left(z_{0}\right)$, we have

$$
G^{\prime}(z(t)) z^{\prime}(t)=3 t^{2} G\left(z_{0}\right), \quad t \in[0,1] .
$$

Using (4.9) and 4.7) we have

$$
\begin{aligned}
\int_{0}^{z_{0}} H_{e^{i \theta}, \lambda}(\zeta) d \zeta-C\left(\lambda, \gamma_{0}\right) & =\int_{0}^{1}\left\{H_{e^{i \theta}, \lambda}(z(t))-c(z(t), \lambda)\right\} z^{\prime}(t) d t \\
& =e^{-i \gamma} \int_{0}^{1} r(z(t), \lambda) \frac{G^{\prime}(z(t)) z^{\prime}(t)}{\left|G^{\prime}(z(t)) z^{\prime}(t)\right|}\left|z^{\prime}(t)\right| d t \\
& =e^{-i \gamma} \frac{G\left(z_{0}\right)}{\left|G\left(z_{0}\right)\right|} \int_{0}^{1} r(z(t), \lambda)\left|z^{\prime}(t)\right| d t \\
& =e^{-i \gamma} \frac{G\left(z_{0}\right)}{\left|G\left(z_{0}\right)\right|} R\left(\lambda, \gamma_{0}\right),
\end{aligned}
$$


where $C\left(\lambda, \gamma_{0}\right)$ and $R\left(\lambda, \gamma_{0}\right)$ are defined as in Corollary 4.3. Thus

$$
\int_{0}^{z_{0}} H_{e^{i \theta}, \lambda}(\zeta) d \zeta \in \partial \overline{\mathbb{D}}\left(C\left(\lambda, \gamma_{0}\right), R\left(\lambda, \gamma_{0}\right)\right) .
$$

Also, from Corollary 4.3 , we have

$$
\int_{0}^{z_{0}} H_{e^{i \theta}, \lambda}(\zeta) d \zeta \in V_{\mathcal{P}}\left(z_{0}, \lambda\right) \subset \overline{\mathbb{D}}\left(C\left(\lambda, \gamma_{0}\right), R\left(\lambda, \gamma_{0}\right)\right) .
$$

Hence, we conclude that $\int_{0}^{z_{0}} H_{e^{i \theta}, \lambda}(\zeta) d \zeta \in \partial V_{\mathcal{P}}\left(z_{0}, \lambda\right)$.

Finally, we prove the uniqueness of the curve. Suppose that

$$
\int_{0}^{z_{0}} P(\zeta) d \zeta=\int_{0}^{z_{0}} H_{e^{i \theta}, \lambda}(\zeta) d \zeta
$$

for some $P \in \mathcal{P}(\lambda)$ and $\theta \in(-\pi, \pi]$. We introduce

$$
h(t)=e^{i \gamma} \frac{\overline{G\left(z_{0}\right)}}{\left|G\left(z_{0}\right)\right|}\{P(z(t))-c(z(t), \lambda)\} z^{\prime}(t),
$$

where $\gamma_{0}: z(t), 0 \leq t \leq 1$, is given by (4.8). Then $h(t)$ is a continuous function in $[0,1]$ and satisfies

$$
|h(t)| \leq r(z(t), \lambda)\left|z^{\prime}(t)\right|
$$

Furthermore, from 4.10 we have

$$
\begin{aligned}
\int_{0}^{1} \operatorname{Re} h(t) d t & =\int_{0}^{1} \operatorname{Re}\left\{e^{i \gamma} \frac{\overline{G\left(z_{0}\right)}}{\left|G\left(z_{0}\right)\right|}\{P(z(t))-c(z(t), \lambda)\} z^{\prime}(t)\right\} d t \\
& =\operatorname{Re}\left\{e^{i \gamma} \frac{\overline{G\left(z_{0}\right)}}{\left|G\left(z_{0}\right)\right|}\left\{\int_{0}^{z_{0}} H_{e^{i \theta}, \lambda}(\zeta) d \zeta-C\left(\lambda, \gamma_{0}\right)\right\}\right\} \\
& =\int_{0}^{1} r(z(t), \lambda)\left|z^{\prime}(t)\right| d t .
\end{aligned}
$$

Thus

$$
h(t)=r(z(t), \lambda)\left|z^{\prime}(t)\right| \quad \text { for all } t \in[0,1] .
$$

From (4.7) and (4.9), it follows that

$$
\int_{0}^{z_{0}} P(\zeta) d \zeta=\int_{0}^{z_{0}} H_{e^{i \theta}, \lambda}(\zeta) d \zeta \quad \text { on } \gamma_{0} .
$$

In view of the identity theorem for analytic functions, we see that it holds for all $z_{0} \in \mathbb{D}$, and hence, by the normalization, $P=H_{e^{i \theta}, \lambda}$ in $\mathbb{D}$. 


\section{Main theorem}

Theorem 5.1. For $\lambda \in \mathbb{D}$ and $z_{0} \in \mathbb{D} \backslash\{0\}$, the boundary $\partial V_{\mathcal{P}}\left(z_{0}, \lambda\right)$ is the Jordan curve given by

$$
\begin{aligned}
(-\pi, \pi] \ni \theta \mapsto & \int_{0}^{z_{0}} H_{e^{i \theta}, \lambda}(\zeta) d \zeta \\
& =\int_{0}^{z_{0}} \frac{1+\left[2(1-\beta)(\cos \gamma) e^{-i \gamma}-1\right] \delta\left(e^{i \theta} \zeta, \lambda\right) \zeta}{1-\delta\left(e^{i \theta} \zeta, \lambda\right) \zeta} d \zeta .
\end{aligned}
$$

If $\int_{0}^{z_{0}} P(\zeta) d \zeta=\int_{0}^{z_{0}} H_{e^{i \theta}, \lambda}(\zeta) d \zeta$ for some $P \in \mathcal{P}(\lambda)$ and $\theta \in(-\pi, \pi]$, then $P(z)=H_{e^{i \theta}, \lambda}(z)$, where $\delta(z, \lambda)$ is defined by $(3.3)$.

Proof. We need to prove that the closed curve

$$
(-\pi, \pi] \ni \theta \mapsto \int_{0}^{z_{0}} H_{e^{i \theta}, \lambda}(\zeta) d \zeta
$$

is simple. Suppose that

$$
\int_{0}^{z_{0}} H_{e^{i \theta_{1}, \lambda}}(\zeta) d \zeta=\int_{0}^{z_{0}} H_{e^{i \theta_{2}, \lambda}}(\zeta) d \zeta
$$

for some $\theta_{1}, \theta_{2} \in(-\pi, \pi]$ with $\theta_{1} \neq \theta_{2}$. Then, from Proposition 4.5, we have

$$
H_{e^{i \theta_{1}, \lambda}}=H_{e^{i \theta_{2}, \lambda}} \text {. }
$$

From (3.5) and (4.4) we obtain the identity

$$
\tau\left(\frac{\omega_{e_{e^{i \theta, \lambda}}}}{z}, \lambda\right)=\frac{e^{i \theta} z\left(1-\bar{\lambda}^{2}\right)+\lambda-\bar{\lambda}}{e^{i \theta} z(\bar{\lambda}-\lambda)+1-\lambda^{2}} .
$$

From $(5.2)$ and $(5.3)$ we have

$$
\frac{e^{i \theta_{1}} z\left(1-\bar{\lambda}^{2}\right)+\lambda-\bar{\lambda}}{e^{i \theta_{1}} z(\bar{\lambda}-\lambda)+1-\lambda^{2}}=\frac{e^{i \theta_{2}} z\left(1-\bar{\lambda}^{2}\right)+\lambda-\bar{\lambda}}{e^{i \theta_{2}} z(\bar{\lambda}-\lambda)+1-\lambda^{2}} .
$$

A simplification of 5.4 gives

$$
e^{i \theta_{1}} z=e^{i \theta_{2}} z,
$$

which is a contradiction to the choice of $\theta_{1}$ and $\theta_{2}$. Thus, the curve must be simple.

Since $V_{\mathcal{P}}\left(z_{0}, \lambda\right)$ is a compact convex subset of $\mathbb{C}$ and has non-empty interior, the boundary $\partial V_{\mathcal{P}}\left(z_{0}, \lambda\right)$ is a simple closed curve. From Proposition 4.1 . the curve $\partial V_{\mathcal{P}}\left(z_{0}, \lambda\right)$ contains the curve (5.1). Recall the fact that a simple closed curve cannot contain any simple closed curve other than itself. Thus, $\partial V_{\mathcal{P}}\left(z_{0}, \lambda\right)$ is given by 5.1). 
REMARK 5.2. The integral in (5.1) can be simplified as follows: Set $b=$ $\operatorname{Im}\left(\bar{\lambda} e^{i \theta / 2}\right) \in \mathbb{R}$. Then a computation shows that

$$
1+\left(\bar{\lambda} e^{i \theta}-\lambda\right) z-e^{i \theta} z^{2}=\left(1-z / z_{1}\right)\left(1-z / z_{2}\right),
$$

where

$$
z_{1}=e^{-i \theta / 2}\left(i b+\sqrt{1-b^{2}}\right) \text { and } z_{2}=e^{-i \theta / 2}\left(i b-\sqrt{1-b^{2}}\right) .
$$

From (3.4) and (3.3) we have

$$
H_{e^{i \theta}, \lambda}(z)=1+2(1-\beta) e^{-i \gamma}(\cos \gamma) \frac{\left(e^{i \theta} z+\lambda\right) z}{1+\left(\bar{\lambda} e^{i \theta}-\lambda\right) z-e^{i \theta} z^{2}} .
$$

Since

$$
\frac{\left(e^{i \theta} z+\lambda\right) z}{1+\left(\bar{\lambda} e^{i \theta}-\lambda\right) z-e^{i \theta} z^{2}}=-1-\frac{e^{-i \theta}}{z_{1}-z_{2}}\left(\frac{1+\bar{\lambda} e^{i \theta} z_{1}}{z-z_{1}}-\frac{1+\bar{\lambda} e^{i \theta} z_{2}}{z-z_{2}}\right),
$$

the equation (5.5) becomes

$$
\begin{aligned}
H_{e^{i \theta}, \lambda}(z)= & -2(1-\beta) e^{-i \gamma} \cos \gamma \\
& -\frac{2 e^{-i \gamma}(1-\beta) e^{-i \theta} \cos \gamma}{z_{1}-z_{2}}\left(\frac{1+\bar{\lambda} e^{i \theta} z_{1}}{z-z_{1}}-\frac{1+\bar{\lambda} e^{i \theta} z_{2}}{z-z_{2}}\right) .
\end{aligned}
$$

By integrating on both sides from 0 to $z_{0}$, we can easily obtain the following representation:

$$
\begin{aligned}
& \int_{0}^{z_{0}} H_{e^{i \theta}, \lambda}(\zeta) d \zeta=\left(1-2(1-\beta) e^{-i \gamma} \cos \gamma\right) z_{0} \\
& \quad+K(\gamma, \beta, \theta, b)\left[\left(1+\bar{\lambda} e^{i \theta / 2}\left(-\sqrt{1-b^{2}}+i b\right)\right) \log \left(1+\frac{e^{i \theta / 2} z_{0}}{\sqrt{1-b^{2}}-i b}\right)\right. \\
& \left.\quad-\left(1+\bar{\lambda} e^{i \theta / 2}\left(\sqrt{1-b^{2}}+i b\right)\right) \log \left(1-\frac{e^{i \theta / 2} z_{0}}{\sqrt{1-b^{2}}+i b}\right)\right],
\end{aligned}
$$

where

$$
K(\gamma, \beta, \theta, b)=\frac{e^{-i \gamma}(1-\beta) e^{-i \theta / 2} \cos \gamma}{\sqrt{1-b^{2}}} .
$$

For $\lambda=0$, Theorem 5.1 takes the following simple form.

Corollary 5.3. For $z_{0} \in \mathbb{D} \backslash\{0\}$ and $\lambda=0$ the boundary $\partial V_{\mathcal{P}}\left(z_{0}, 0\right)$ is the Jordan curve given by

$$
\begin{aligned}
(-\pi, \pi] & \ni \theta \mapsto \int_{0}^{z_{0}} H_{e^{i \theta}, 0}(\zeta) d \zeta \\
& =\left(1-2(1-\beta) e^{-i \gamma} \cos \gamma\right) z_{0}+e^{-i \gamma}(1-\beta) e^{-i \theta / 2} \cos \gamma \log \frac{1+e^{i \theta / 2} z_{0}}{1-e^{i \theta / 2} z_{0}} .
\end{aligned}
$$


If $\int_{0}^{z_{0}} P(\zeta) d \zeta=\int_{0}^{z_{0}} H_{e^{i \theta}, 0}(\zeta) d \zeta$ for some $P \in \mathcal{P}(0)$ and $\theta \in(-\pi, \pi]$, then $P(z)=H_{e^{i \theta}, 0}(z)$.

\section{Some special cases}

6.1. The class $\mathcal{R}_{\beta}$. In order to discuss a special situation, we consider $P=f^{\prime}$ and $\gamma=0$ in the class $\mathcal{P}_{\gamma, \beta}$. Thus, $\mathcal{P}_{\gamma, \beta}$ reduces to $\mathcal{R}_{\beta}$, where

$$
\mathcal{R}_{\beta}=\left\{f \in \mathcal{A}: \operatorname{Re} f^{\prime}(z)>\beta \text { in } \mathbb{D}\right\} .
$$

Then $\mathcal{R}_{\beta} \subset \mathcal{S}$ for $0 \leq \beta<1$. As with $\mathcal{P}(\lambda)$, for $\lambda \in \overline{\mathbb{D}}$ and $z_{0} \in \mathbb{D}$ being fixed, we define

$$
\mathcal{R}(\lambda)=\left\{f \in \mathcal{R}_{\beta}: f^{\prime \prime}(0)=2(1-\beta) \lambda\right\}, \quad V_{\mathcal{R}}\left(z_{0}, \lambda\right)=\left\{f\left(z_{0}\right): f \in \mathcal{R}(\lambda)\right\} .
$$

We remark that if $f \in \mathcal{R}(\lambda)$, then necessarily

$$
f^{\prime \prime \prime}(0)=4(1-\beta)\left[\left(1-|\lambda|^{2}\right) a+\lambda^{2}\right]
$$

for some $a \in \overline{\mathbb{D}}$.

For $P=f^{\prime}$, a computation shows that the extremal function $H_{e^{i \theta}, \lambda}(z)$ for the class $\mathcal{R}(\lambda)$ takes the form

$$
H_{e^{i \theta}, \lambda}(z)=z_{0}+2(1-\beta) \int_{0}^{z_{0}} \frac{\left(e^{i \theta} \zeta+\lambda\right) \zeta}{1+\bar{\lambda} e^{i \theta} \zeta-\left(e^{i \theta} \zeta+\lambda\right) \zeta} d \zeta .
$$

It is not difficult to obtain the following result, which is the analog of Theorem 5.1 for the class $\mathcal{R}(\lambda)$.

Corollary 6.1. For $\lambda \in \mathbb{D}$ and $z_{0} \in \mathbb{D} \backslash\{0\}$, the boundary $\partial V_{\mathcal{R}}\left(z_{0}, \lambda\right)$ is the Jordan curve given by

$$
(-\pi, \pi] \ni \theta \mapsto H_{e^{i \theta}, \lambda}\left(z_{0}\right)=z_{0}+2(1-\beta) \int_{0}^{z_{0}} \frac{\left(e^{i \theta} \zeta+\lambda\right) \zeta}{1+\bar{\lambda} e^{i \theta} \zeta-\left(e^{i \theta} \zeta+\lambda\right) \zeta} d \zeta .
$$

If $f\left(z_{0}\right)=H_{e^{i \theta}, \lambda}\left(z_{0}\right)$ for some $f \in \mathcal{R}(\lambda)$ and $\theta \in(-\pi, \pi]$, then $f(z)=$ $H_{e^{i \theta}, \lambda}(z)$.

For $0 \leq \beta<1$ and $\lambda=0$, set

$$
\mathcal{R}(0)=\left\{f \in \mathcal{A}: f^{\prime \prime}(0)=0 \text { and } \operatorname{Re} f^{\prime}(z)>\beta \text { in } \mathbb{D}\right\} \subset \mathcal{R}_{\beta} .
$$

In particular, the choices $\gamma=0$ and $P(z)=f^{\prime}(z)$ in Corollary 4.2 give the following: if $f \in \mathcal{R}(0) \subset \mathcal{R}_{\beta}$ for some $0 \leq \beta<1 / 2$, then by (4.6), one has

$$
\left|f^{\prime}(z)\right| \leq \frac{1+(1-2 \beta)|z|^{4}+2(1-\beta)|z|^{2}}{1-|z|^{4}}=\frac{1+(1-2 \beta)|z|^{2}}{1-|z|^{2}}, \quad z \in \mathbb{D},
$$

so that

$$
\sup _{z \in \mathbb{D}}\left(1-|z|^{2}\right)\left|f^{\prime}(z)\right| \leq 2(1-\beta)
$$


Equality holds for

$$
f(z)=\beta z+\frac{1-\beta}{2} \log \frac{1+z}{1-z}, \quad z \in \mathbb{D} .
$$

6.2. The class $\mathcal{F}(\alpha, \beta)$. For $\alpha \in \mathbb{C}$ satisfying $\operatorname{Re} \alpha>0$ and $\beta \in \mathbb{R}$ with $\beta<1$, let $\mathcal{F}(\alpha, \beta)$ denote the class of functions $f \in \mathcal{A}$ satisfying

$$
f^{\prime}(z)+\alpha z f^{\prime \prime}(z) \prec \frac{1+(1-2 \beta) z}{1-z}, \quad z \in \mathbb{D},
$$

where $\prec$ denotes the usual subordination [MM]. In [Po1] conditions on $\alpha$ and $\beta$ for which

$$
\mathcal{F}(\alpha, \beta) \subset \mathcal{S}^{*}
$$

have been established (also for certain complex values of $\alpha$ ), and in [FR] it has been shown that $\mathcal{F}(\alpha, \beta) \subset \mathcal{S}^{*}$ if $\alpha \geq 1 / 3$ and $\beta \geq \beta_{0}(\alpha)$, where

$$
\beta_{0}(\alpha)=\frac{-\frac{1}{\alpha} \int_{0}^{1} t^{1 / \alpha-1} \frac{1+t}{1-t} d t}{1-\frac{1}{\alpha} \int_{0}^{1} t^{1 / \alpha-1} \frac{1+t}{1-t} d t} .
$$

This is indeed a reformulated version of a theorem from [FR and the inclusion is sharp in the following sense: for $\beta<\beta_{0}(\alpha)$ the functions in $\mathcal{F}(\alpha, \beta)$ are not even univalent in $\mathbb{D}$. For an extension of this inclusion result, we refer to [PR1, PR2].

Now, we present an alternative representation for functions in $\mathcal{F}(\alpha, \beta)$. If $f \in \mathcal{F}(\alpha, \beta)$, then 6.1 is equivalent to

$$
\frac{f(z)}{z} *\left(1+\sum_{n=2}^{\infty} n(1+(n-1) \alpha) z^{n-1}\right) \prec 1+2(1-\beta) \frac{z}{1-z},
$$

where $*$ denotes the Hadamard product (or convolution) of two analytic functions in $\mathbb{D}$ represented by power series about the origin. By a well-known convolution theorem (cf. [RS] ) this gives

$$
\frac{f(z)}{z} \prec \beta+(1-\beta)\left[1+\frac{2}{\alpha} \sum_{n=1}^{\infty} \frac{z^{n}}{(n+1)(n+1 / \alpha)}\right]
$$

and a computation shows that

$$
\frac{f(z)}{z} \prec\left\{\begin{array}{lr}
\beta+(1-\beta)\left[1-\frac{2}{1-\alpha}\left(\frac{\log (1-z)}{z}+1+\int_{0}^{1} t^{1 / \alpha} \frac{z}{1-t z} d t\right)\right] \\
\beta+(1-\beta)\left[1+2 z \int_{0}^{1} \frac{t \log (1 / t)}{1-t z} d t\right] & \text { if } \alpha \neq 1,
\end{array}\right.
$$


The definition of subordination gives the following representation of functions in $\mathcal{F}(\alpha, \beta)$ :

$$
f(z)=\left\{\begin{array}{lr}
z-\frac{2(1-\beta) z}{1-\alpha}\left\{1+\frac{1}{\omega(z)} \log (1-\omega(z))+\omega(z) \int_{0}^{1} \frac{t^{1 / \alpha}}{1-t \omega(z)} d t\right\} \\
z+2(1-\beta) z \omega(z) \int_{0}^{1} \frac{t \log (1 / t)}{1-t \omega(z)} d t & \text { if } \alpha \neq 1,
\end{array}\right.
$$

for $z \in \mathbb{D}$, and for some $\omega \in \mathcal{B}_{0}$.

If $f \in \mathcal{F}(\alpha, \beta)$, then according to the Herglotz representation there exists a unique positive unit measure $\mu$ on $(-\pi, \pi]$ such that

$$
f^{\prime}(z)+\alpha z f^{\prime \prime}(z)=\int_{-\pi}^{\pi} \frac{1+(1-2 \beta) z e^{-i t}}{1-z e^{-i t}} d \mu(t)
$$

or equivalently

$$
\frac{f(z)}{z}=\left[1+\frac{1}{\alpha} \sum_{n=1}^{\infty} \frac{z^{n}}{(n+1)(n+1 / \alpha)}\right] * \int_{-\pi}^{\pi} \frac{1+(1-2 \beta) z e^{-i t}}{1-z e^{-i t}} d \mu(t) .
$$

A simplification of the last equality gives the following representation of functions in the class $\mathcal{F}(\alpha, \beta)$ :

$$
f(z)=\left\{\begin{array}{cc}
\frac{z}{1-\alpha} \int_{0-\pi}^{1} \int_{-\pi}^{\pi}\left(1-s^{1 / \alpha-1}\right)\left(\frac{1+(1-2 \beta) s z e^{-i t}}{1-s z e^{-i t}}\right) d \mu(t) d s \\
\quad \text { if } \alpha \neq 1, \\
z+2(1-\beta) z \int_{0-\pi}^{1} \int_{-\pi}^{\pi}\left(\log \frac{1}{s}\right)\left(\frac{s z e^{-i t}}{1-s z e^{-i t}}\right) d \mu(t) d s & \text { if } \alpha=1 .
\end{array}\right.
$$

To state our special case in precise form, for convenience we let $\gamma=0$, and define

$$
P(z)=f^{\prime}(z)+\alpha z f^{\prime \prime}(z), \quad f \in \mathcal{F}(\alpha, \beta),
$$

so that

$$
P^{\prime}(0)=(1+\alpha) f^{\prime \prime}(0) \quad \text { and } \quad P^{\prime \prime}(0)=(1+2 \alpha) f^{\prime \prime}(0) .
$$

In view of these observations, the analogs of the sets $\mathcal{P}(\lambda)$ and $V_{\mathcal{P}}\left(z_{0}, \lambda\right)$ will be

$$
\begin{aligned}
\mathcal{G}(\lambda) & =\left\{f \in \mathcal{F}(\alpha, \beta): f^{\prime \prime}(0)=2 \frac{1-\beta}{1+\alpha} \lambda\right\}, \\
V_{\mathcal{G}}\left(z_{0}, \lambda\right) & =\left\{(1-\alpha) f\left(z_{0}\right)+\alpha z_{0} f^{\prime}\left(z_{0}\right): f \in \mathcal{G}(\lambda)\right\},
\end{aligned}
$$


where $0 \leq \beta<1$. We observe that for functions $f$ in $\mathcal{G}(\lambda)$,

$$
f^{\prime \prime \prime}(0)=4\left(\left(1-|\lambda|^{2}\right) a+\lambda^{2}\right) \frac{1-\beta}{1+2 \alpha}
$$

for some $a \in \overline{\mathbb{D}}$.

With $P(z)=f^{\prime}(z)+\alpha z f^{\prime \prime}(z)$, the corresponding extremal function $F_{e^{i \theta}, \lambda}(z)$ for $\mathcal{G}(\lambda)$ can be computed to be

$$
(1-\alpha) F_{e^{i \theta}, \lambda}(z)+\alpha z F_{e^{i \theta}, \lambda}^{\prime}(z)=\int_{0}^{z} \frac{1+(1-2 \beta) \delta(a \zeta, \lambda) \zeta}{1-\delta(a \zeta, \lambda) \zeta} d \zeta
$$

where $\delta(z, \lambda)$ is defined by (3.3). In this setting, Proposition 4.1 (for $\gamma=0$ ) takes the following form:

Proposition 6.2. For $f \in \mathcal{G}(\lambda)$ and $\lambda \in \mathbb{D}$, we have

$$
\left|f^{\prime}(z)+\alpha z f^{\prime \prime}(z)-c(z, \lambda)\right| \leq r(z, \lambda), \quad z \in \mathbb{D},
$$

where

$$
\begin{aligned}
& c(z, \lambda)=\frac{(1+(1-2 \beta) \lambda z)(1-\bar{\lambda} \bar{z})+|z|^{2}(\bar{z}-\lambda)(\bar{\lambda}+(1-2 \beta) z)}{\left(1-|z|^{2}\right)\left(1+|z|^{2}-2 \operatorname{Re}(\lambda z)\right)}, \\
& r(z, \lambda)=\frac{2(1-\beta)\left(1-|\lambda|^{2}\right)|z|^{2}}{\left(1-|z|^{2}\right)\left(1+|z|^{2}-2 \operatorname{Re}(\lambda z)\right)} .
\end{aligned}
$$

For each $z \in \mathbb{D} \backslash\{0\}$, equality holds if and only if $f=F_{e^{i \theta}, \lambda}$ for some $\theta \in \mathbb{R}$.

Using Theorem 5.1, we get the following result.

Corollary 6.3. For $\lambda \in \mathbb{D}, z_{0} \in \mathbb{D} \backslash\{0\}$ and $\alpha \in \mathbb{C}$ with $\operatorname{Re} \alpha>0$, the boundary $\partial V_{\mathcal{G}}\left(z_{0}, \lambda\right)$ is the Jordan curve given by

$$
\begin{aligned}
(-\pi, \pi] & \ni \theta \mapsto(1-\alpha) F_{e^{i \theta}, \lambda}\left(z_{0}\right)+\alpha z_{0} F_{e^{i \theta, \lambda}}^{\prime}\left(z_{0}\right)=(2 \beta-1) z_{0} \\
+ & \frac{(1-\beta) e^{-i \theta / 2}}{\sqrt{1-b^{2}}}\left[\left(1+\bar{\lambda} e^{i \theta / 2}\left(-\sqrt{1-b^{2}}+i b\right)\right) \log \left(1+\frac{e^{i \theta / 2} z_{0}}{\sqrt{1-b^{2}}-i b}\right)\right. \\
& \left.-\left(1+\bar{\lambda} e^{i \theta / 2}\left(\sqrt{1-b^{2}}+i b\right)\right) \log \left(1-\frac{e^{i \theta / 2} z_{0}}{\sqrt{1-b^{2}}+i b}\right)\right],
\end{aligned}
$$

where $b=\operatorname{Im}\left(\bar{\lambda} e^{i \theta / 2}\right)$. If $(1-\alpha) f\left(z_{0}\right)+\alpha z_{0} f^{\prime}\left(z_{0}\right)=(1-\alpha) F_{a, \lambda}\left(z_{0}\right)+$ $\alpha z_{0} F_{a, \lambda}^{\prime}\left(z_{0}\right)$ for some $f \in \mathcal{G}(\lambda)$ and $\theta \in(-\pi, \pi]$, then $f(z)=F_{e^{i \theta}, \lambda}(z)$.

The proof of this corollary follows by taking $\gamma=0$ in Remark 5.2 and so we omit the details.

In the case of $\lambda=0$ in Corollary 6.3, the corresponding extremal function $F_{a, 0}(z)$ can be obtained easily by solving

$$
F_{a, 0}^{\prime}(z)+\alpha z F_{a, 0}^{\prime \prime}(z)=\frac{1+(1-2 \beta) \delta(a z, 0) z}{1-\delta(a z, 0) z} .
$$


This may be rewritten as

$$
\frac{F_{a, 0}(z)}{z} *\left[1+\sum_{n=0}^{\infty}(n+1)(1+n \alpha) z^{n}\right]=2 \beta-1+2(1-\beta) \frac{1}{1-a z^{2}}
$$

or equivalently as

$$
\frac{F_{a, 0}(z)}{z}=\left[1+\sum_{n=1}^{\infty} 2(1-\beta) a^{n} z^{2 n}\right] *\left[1+\sum_{n=0}^{\infty} \frac{z^{n}}{(n+1)(1+n \alpha)}\right] .
$$

A simple calculation gives

$$
F_{a, 0}(z)= \begin{cases}z+\frac{(1-\beta) a z^{3}}{1-\alpha} \int_{0}^{1} \frac{t^{1 / 2}-t^{1 / 2 \alpha}}{1-t a z^{2}} d t & \text { if } \alpha \neq 1 \\ z+\frac{(1-\beta) a z^{3}}{2} \int_{0}^{1} \frac{t^{1 / 2} \log (1 / t)}{1-t a z^{2}} d t & \text { if } \alpha=1\end{cases}
$$

7. Geometric view of Theorem 5.1. Using Mathematica (see $[\mathrm{R}]$ ), we describe the boundary of the sets $V_{\mathcal{P}}\left(z_{0}, \lambda\right)$ and $V_{\mathcal{G}}\left(z_{0}, \lambda\right)$. In the program below, "z0" stands for $z_{0}$, "lam" for $\lambda$, "g" for $\gamma$ and "b" for $\beta$.

(* Geometric view of the main Theorem 5.1 and Corollary $6.3 *$ )

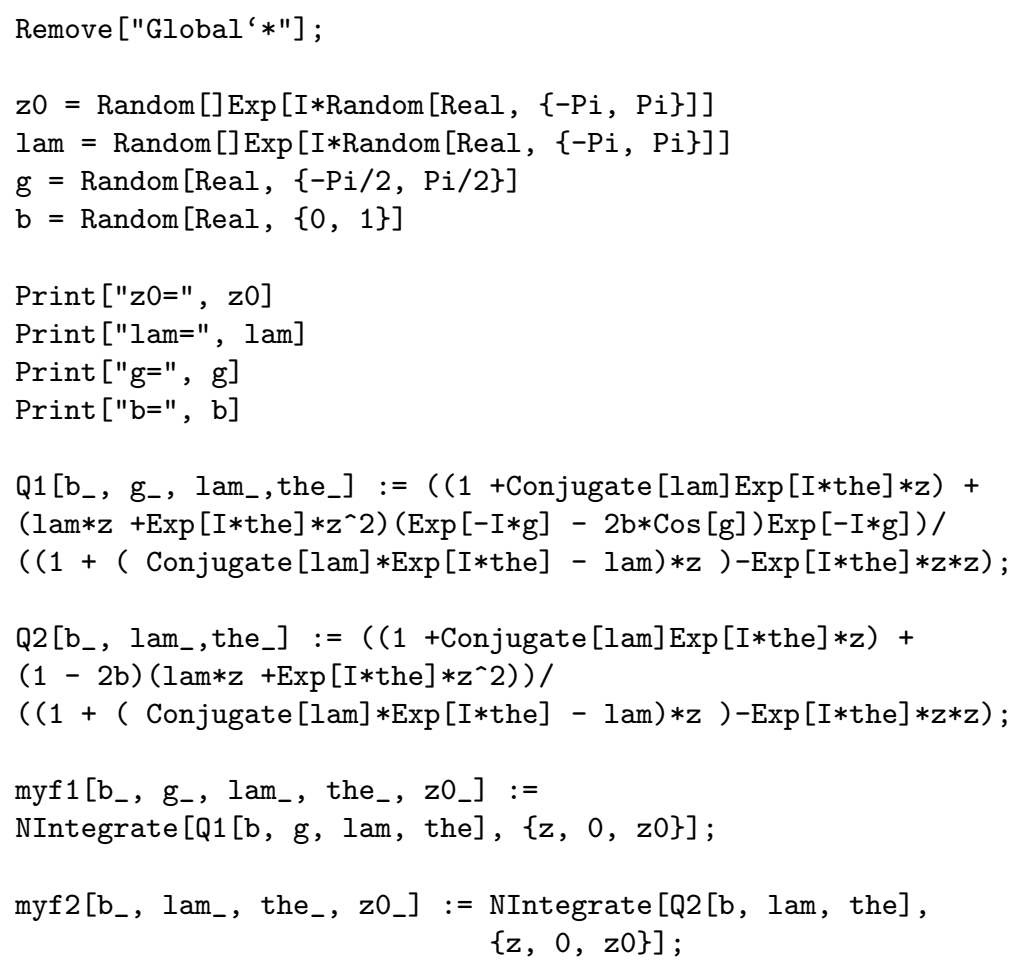




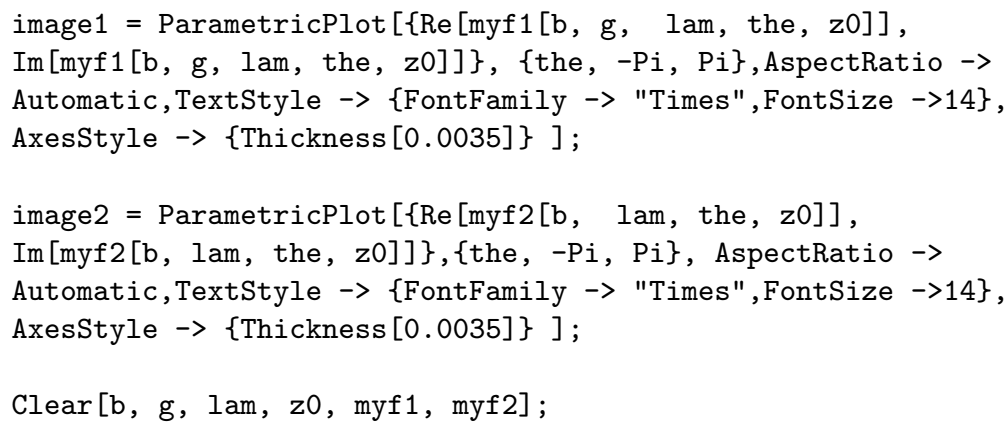

The following figures show the boundary of $V_{\mathcal{P}}\left(z_{0}, \lambda\right)$ and $V_{\mathcal{G}}\left(z_{0}, \lambda\right)$ for certain values of $z_{0} \in \mathbb{D} \backslash\{0\}, \lambda \in \mathbb{D}, 0 \leq \beta<1$ and $|\gamma|<\pi / 2$. Table 1 gives the list of these parameter values corresponding to Figs. $1-5$. We recall that according to Proposition 3.1 the region bounded by the curve $\partial V_{\mathcal{P}}\left(z_{0}, \lambda\right)$ is compact and convex.

\section{Table 1}

\begin{tabular}{ccccc}
\hline Fig. & $z_{0}$ & $\lambda$ & $\beta$ & $\gamma$ \\
\hline 1 & $0.335192-0.787333 i$ & $0.0737292+0.466706 i$ & 0.591244 & 0.383292 \\
2 & $-0.261209+0.926935 i$ & $-0.28588+0.307498 i$ & 0.700318 & -0.87825 \\
3 & $-0.41227-0.521734 i$ & $-0.0875648+0.0714166 i$ & 0.602203 & 0.910581 \\
4 & $0.771264+0.151204 i$ & $-0.391149-0.294747 i$ & 0.928608 & 1.55854 \\
5 & $0.335626+0.929093 i$ & $0.00010443+0.0255256 i$ & 0.76622 & 1.5449 \\
\hline
\end{tabular}

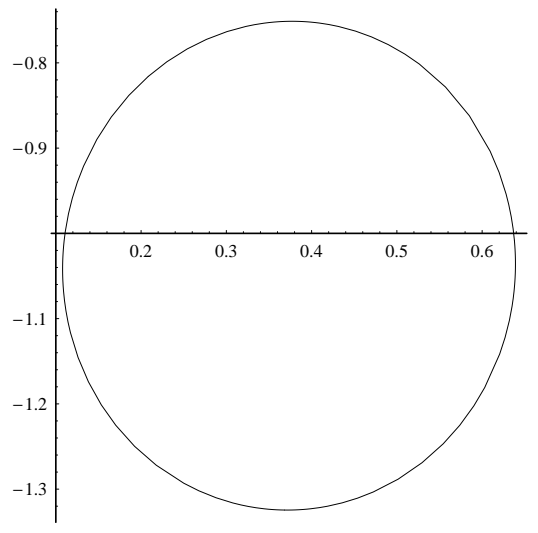

$\partial V_{\mathcal{P}}\left(z_{0}, \lambda\right)$

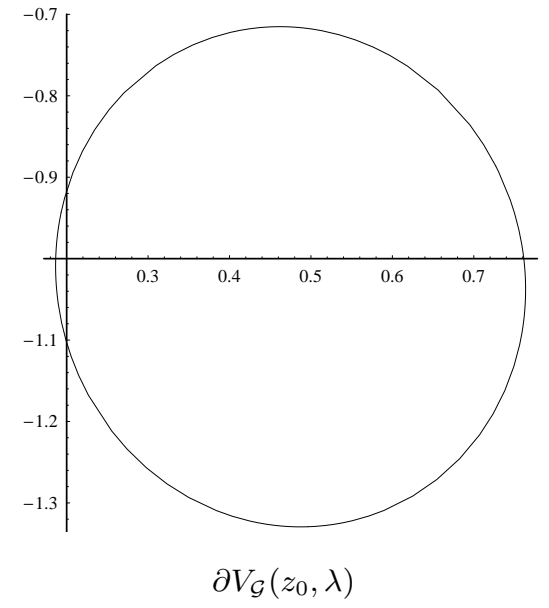

$\partial V_{\mathcal{G}}\left(z_{0}, \lambda\right)$

Fig. 1. $z_{0}=0.335192-0.787333 i$ and $\beta=0.591244$ 


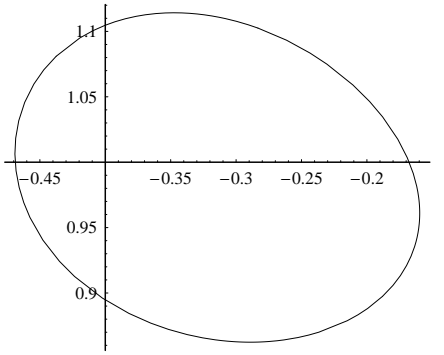

$$
\partial V_{\mathcal{P}}\left(z_{0}, \lambda\right)
$$

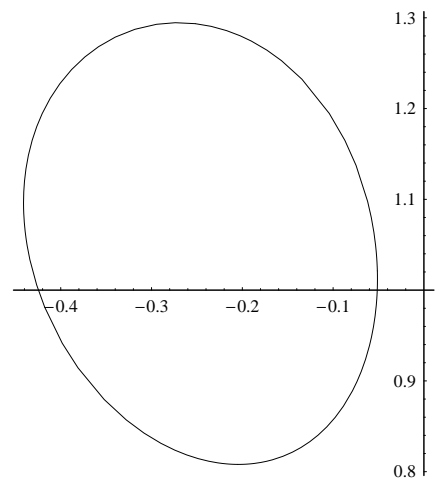

$\partial V_{\mathcal{G}}\left(z_{0}, \lambda\right)$

Fig. 2. $z_{0}=-0.261209+0.926935 i$ and $\beta=0.700$

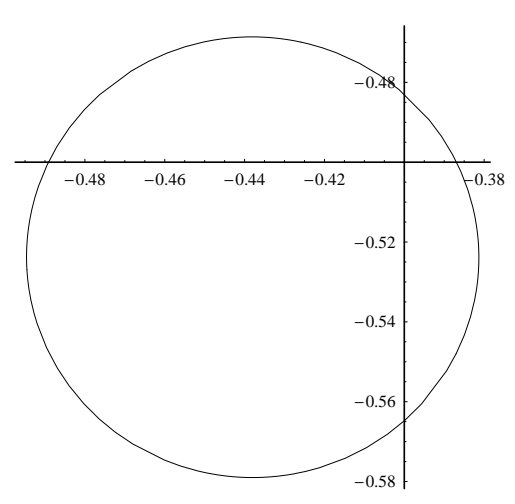

$\partial V_{\mathcal{P}}\left(z_{0}, \lambda\right)$

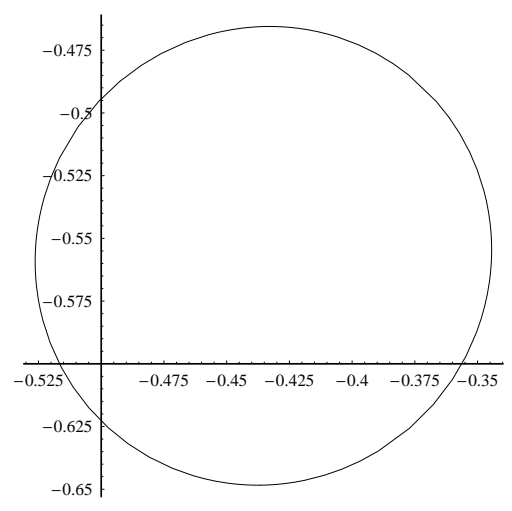

$\partial V_{\mathcal{G}}\left(z_{0}, \lambda\right)$

Fig. 3. $z_{0}=-0.41227-0.521734 i$ and $\beta=0.602203$
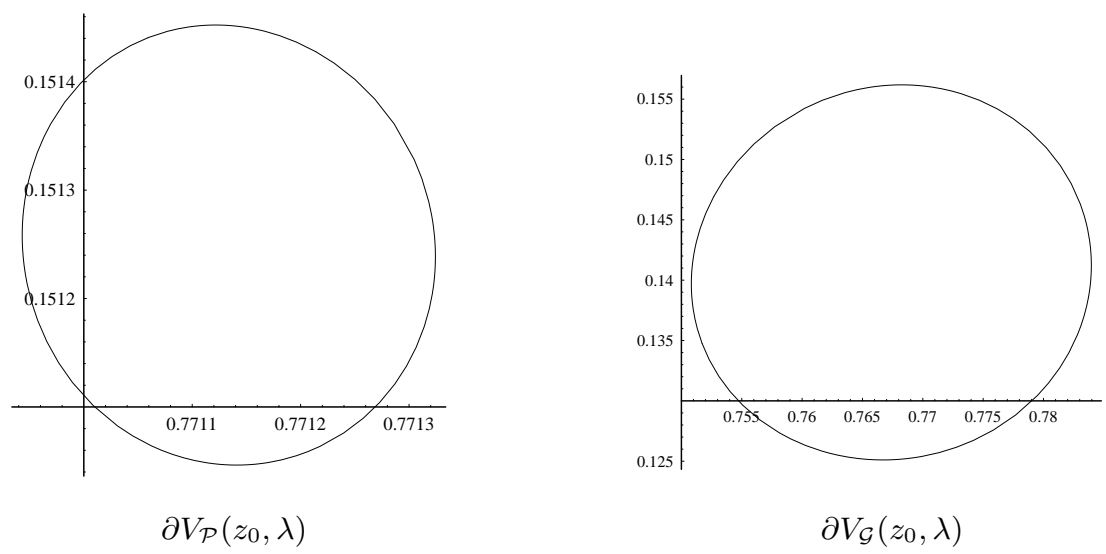

Fig. 4. $z_{0}=0.771264+0.151204 i$ and $\beta=0.928608$ 

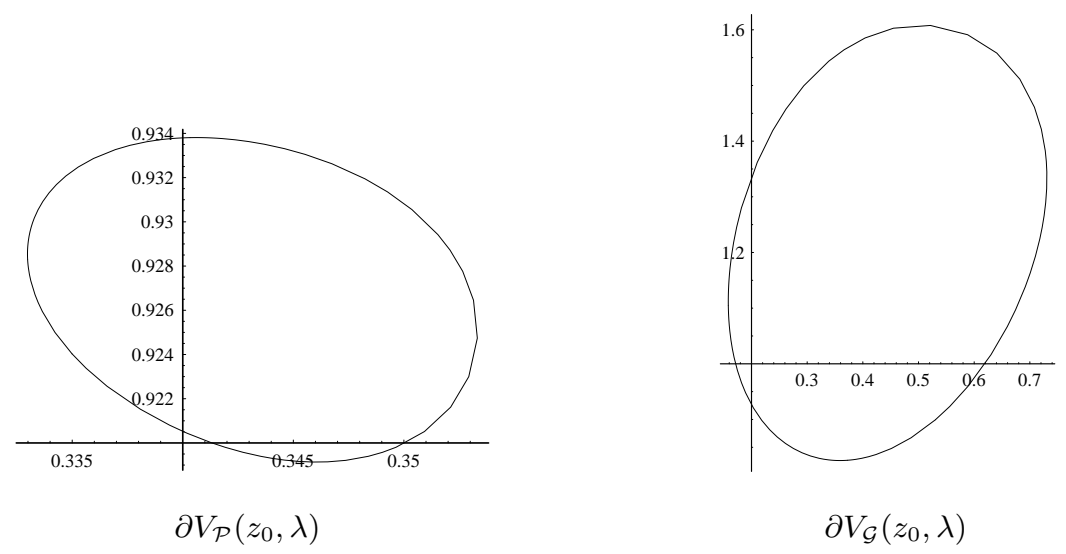

Fig. 5. $z_{0}=0.335626+0.929093 i$ and $\beta=0.76622$

Acknowledgments. This research was supported by National Board for Higher Mathematics (DAE, India; grant no. 48/2/2006/R\&D-II).

\section{References}

[Di] S. Dineen, The Schwarz Lemma, Oxford Math. Monogr., Clarendon Press, Oxford, 1989.

[Du] P. L. Duren, Univalent Functions, Grundlehren Math. Wiss. 259, Springer, New York, 1983.

[FR] R. Fournier and S. Ruscheweyh, On two extremal problems related to univalent functions, Rocky Mountain J. Math. 24 (1994), 529-538.

[MM] S. S. Miller and P. T. Mocanu, Differential Subordinations: Theory and Applications, Dekker, New York, 2000.

[Pa] V. Paatero, Über die konforme Abbildung von Gebieten, deren Ränder von beschränkter Drehung sind, Ann. Acad. Sci. Fenn. Ser. A 33 (1931), no. 9, 1-78.

[Pi] B. Pinchuk, Extremal problems in the class of close-to-convex functions, Trans. Amer. Math. Soc. 129 (1967), 466-478.

[Po1] S. Ponnusamy, Differential subordinations concerning starlike functions, Proc. Indian Acad. Sci. Math. Sci. 104 (1994), 397-411.

[Po2] - Foundations of Complex Analysis, Alpha Science International, 2005.

[PR1] S. Ponnusamy and F. Rønning, Duality for Hadamard products applied to certain integral transforms, Complex Variables Theory Appl. 32 (1997), 263-287.

[PR2] - - - Integral transforms of functions with the derivative in a halfplane, Israel J. Math. 144 (1999), 177-188.

[PS] S. Ponnusamy and H. Silverman, Complex Variables with Applications, Birkhäuser, Boston, 2006.

[PV] S. Ponnusamy and A. Vasudevarao, Region of variability of two subclasses of univalent functions, J. Math. Anal. Appl. 332 (2007), 1323-1334.

[PVV1] S. Ponnusamy, A. Vasudevarao and M. Vuorinen, Region of variability for spirallike functions with respect to a boundary point, Colloq. Math. 116 (2009), 31-46.

[PVV2] - - - - , Region of variability for certain classes of univalent functions satisfying differential inequalities, Complex Var. Elliptic Equations 54 (2009), 899-922. 
[PVY1] S. Ponnusamy, A. Vasudevarao and H. Yanagihara, Region of variability of univalent functions $f(z)$ for which $z f^{\prime}(z)$ is spirallike, Houston J. Math. 34 (2008), 1037-1048.

[PVY2] - - - - Region of variability for close-to-convex functions, Complex Var. Elliptic Equations 53 (2008), 709-716.

[RS] St. Ruscheweyh and J. Stankiewicz, Subordination under convex univalent functions, Bull. Polish Acad. Sci. Math. 33 (1985), 499-502.

[R] H. Ruskeepää, Mathematica Navigator: Mathematics, Statistics, and Graphics, 2nd ed., Elsevier, Burlington, MA, 2004.

[S] L. Špaček, Contribution à la théorie des fonctions univalentes, Časopis Pst. Mat. Fys. 62 (1932), 12-19 (in Czech, with French summary).

[Y1] H. Yanagihara, Regions of variability for functions of bounded derivatives, Kodai Math. J. 28 (2005), 452-462.

[Y2] - Regions of variability for convex function, Math. Nachr. 279 (2006), 17231730.

Saminathan Ponnusamy, Allu Vasudevarao

Department of Mathematics

Indian Institute of Technology Madras

Chennai 600 036, India

E-mail: samy@iitm.ac.in

alluvasudevarao@gmail.com

Received 3.8.2009

and in final form 27.12.2009 
